\title{
Incisional Hernia After Laparoscopic-Assisted Right Hemicolectomy
}

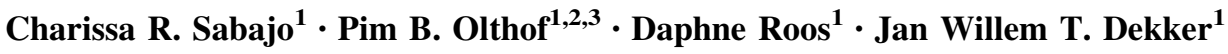

Published online: 19 August 2019

(c) The Author(s) 2019

\begin{abstract}
Background Different approaches used for laparoscopic right colectomy have different advantages and disadvantages. This study aims to determine the incidence and clinical relevance of IH after LARHC as the preferred technique in an experienced setting and to assess which factors are correlated with the development of IH.

Methods Between January 2012 and December 2016, all consecutive patients who underwent LARHC were included. Data were obtained in accordance with the Dutch ColoRectal Audit, and IH was scored based on physical examination and imaging at standard follow-up. Logistic regression analysis was used to identify risk factors for IH. Results A total of 170 patients underwent LARHC. In the same period, 64 patients had an open RHC. IH after LARHC was seen in 24 patients after a median time of 7 months (14\%). Only four of these patients underwent operative IH repair (2\%). Interestingly, a trend for more IH was seen between two surgeons. Multivariable analysis identified BMI [OR 1.08 (95\% CI 1.00-1.15) $P=0.043$ ], a history of smoking [OR 2.14 (95\% CI 1.03-4.41) $P=0.040$ ], and surgical site infection [OR 2.99 (95\% CI 1.28-7.00) $P=0.012$ ] as risk factors for IH.

Conclusion IH incidence after LARHC was considerable, but few were clinically relevant IHs. The IH incidence should be included in shared decision making. The low clinically relevant IH rate does in our opinion not outweigh possible advantages of LARHC.
\end{abstract}

\section{Introduction}

In many centers, the laparoscopic approach has become the standard for colon cancer surgery. In several studies, laparoscopy was shown to result in less morbidity and a shorter hospital stay than an open approach [1-5], without

Pim B. Olthof

p.b.olthof@amc.nl

1 Department of Surgery, Reinier de Graaf Gasthuis, Reinier de Graafweg 5, 2625 AD Delft, The Netherlands

2 Department of Surgery, Erasmus Medical Center, Erasmus University, Rotterdam, The Netherlands

3 Department of Surgery, Amsterdam University Medical Center, University of Amsterdam, Amsterdam, The Netherlands concessions to tumor recurrence, survival, and quality of life [2, 6-8].

Laparoscopic procedures for right hemicolectomy (RHC) can be classified based on the amount of the procedure performed laparoscopically and on the site of anastomosis creation [9-12]. Laparoscopy-assisted RHC (LARHC) involves laparoscopic mobilization of the right hemicolon after which an extracorporeal anastomosis is performed. When using the laparoscopic RHC (LRHC), the procedure is performed entirely laparoscopic with an intracorporeal anastomosis. In LRHC, the skin incisions can be left even smaller and the extraction site is not bound to location limitations of colon extraction [9-12].

To date, none of the laparoscopic techniques has been identified as definitively superior to others. Some studies suggest a shorter operative time and lower conversions 
rates in LARHC compared to LRHC, and most studies did not find any significant differences $[9,10]$. The benefits of extra- over intra-corporeal anastomosis are equally ambiguous, although the most recent meta-analysis did show less short-term morbidity when using intra-corporeal anastomoses; however, a complete laparoscopic approach is technically more demanding.

The differences between techniques in longer-term morbidity, primarily incisional hernia (IH), are also unclear. Reported rates of IH following LARHC vary from 4 to $21 \%$ in mostly small series. IH rates vary from 2 to $6 \%$ following intra-corporal anastomosis which could be due to alternative extraction sites such as a Pfannenstiel incision [13], but again these series are small with only a handful of events [12]. In addition, these studies usually come from centers that perform both techniques and are not dedicated to a single approach; therefore, the exact IH rates in experienced hands are unclear, which limits the comparison of laparoscopic techniques.

This study aimed to investigate the incisional hernia rate following standardized LARHC with extracorporeal anastomosis in an unselected cohort and identify risk factors for incisional hernia.

\section{Methods}

All consecutive patients who underwent LARHC between January 2012 and December 2016 at the Reinier de Graaf Gasthuis, the Netherlands, were included in this study. As a reference for possible selection, data on patients who underwent open RHC were also collected. Data were obtained from a prospective database, which was gathered in accordance with the Dutch ColoRectal Cancer Audit [14]. Additional data were collected from the electronic medical records. The need for ethical approval and individual informed consent was waived by the institutional medical ethics committee.

\section{Patient work up and surgery}

All elective patients were preoperatively discussed at a multidisciplinary meeting. Routine work up included an abdominal CT scan, thoracic X-ray, and baseline carcinoembryonic antigen (CEA) level. All patients were considered for laparoscopic surgery, except for emergency cases with bowel obstruction, patients with preoperatively anticipated T4 tumors, or high-risk patients due to previous abdominal surgery. The laparoscopic-assisted approach was the standard laparoscopic approach, and patients with an occasional total laparoscopic procedure were excluded from the analysis. Patients were operated in supine position, and open instruction was performed using an infra- umbilical incision. Three additional trocards were used (two $5 \mathrm{~mm}$ and one $10 \mathrm{~mm}$ ) The lateral to medial approach was used for all cases (Fig. 1). Extracorporeal hand-sewn anastomosis and specimen extraction were performed using a midline umbilical incision with a maximal length of $5 \mathrm{~cm}$ starting from the infra-umbilical trocard.

\section{Primary outcome}

Data on IH were collected and recorded as present when observed during follow-up by the surgeon or nurse practitioner, or at any other hospital visit. Furthermore, all radiological abdominal diagnostics (CT scan or ultrasound) were reviewed for evidence of a possible asymptomatic IH. Incisional hernias were scored according to the symptoms and treatment and classified according to the European Hernia Society classification for incisional hernias [15].

\section{Variables}

Comorbidity was defined as the presence of any of the following: cardiovascular disease, pulmonary disease, neurologic disease, endocrinology disease (diabetes mellitus and any thyroid problem), renal disease, ulcer disease, Crohn's disease, or colitis.

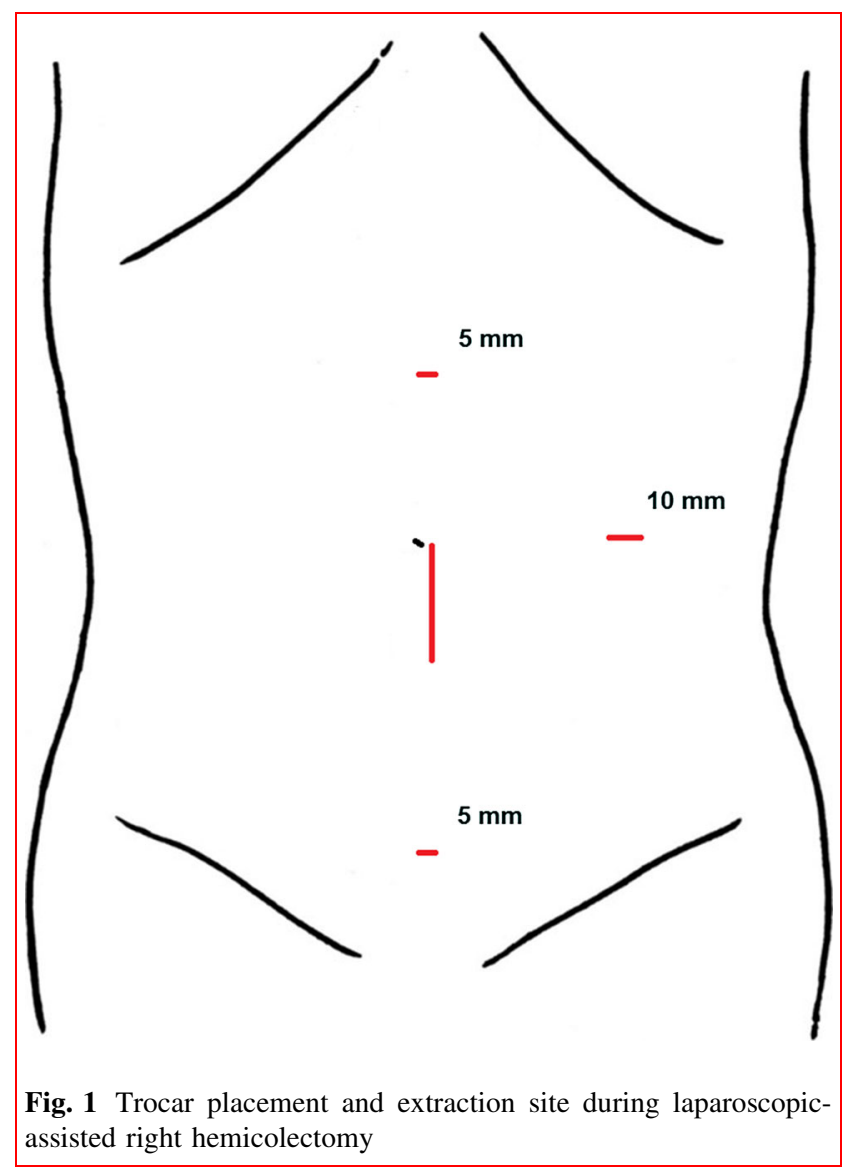


All complications within 30 days after surgery were scored and graded according to Dindo et al. [16] with complications of grade IIIa or higher considered as a major complication. In addition, the comprehensive complications index (CCI) was calculated for each patient [17]. Conversion was defined as an unplanned incision over 5 $\mathrm{cm}$ and was always performed using a midline infra-umbilical incision.

\section{Statistical analysis}

Continuous data were presented as median with interquartile range (IQR). Categorical variables were analyzed using the Chi-square or Fishers' exact tests, and continues variables were analyzed using Mann-Whitney $U$ test. Uni- and multivariable analyses were performed using logistic regression analysis. Variables with $\mathrm{p}$ values equal to or below 0.100 in the univariable analysis were included in multivariable analysis. A $P$ value below 0.05 was considered to indicate statistical significance.

Data analyses were performed using SPSS (version 24, IBM, Chicago, IL).

\section{Results}

In the study period, a total of 254 patients underwent RHC. Of these patients, LARHC was performed in 170 patients and 64 patients underwent open RHC. The remaining 20 patients underwent LRHC and were excluded from the analysis.

Baseline patients and disease characteristics as well as outcomes are displayed in Table 1.

The laparoscopic approach was the standard treatment, and the open approach was reserved for emergency cases, patients with anticipated T4 tumors, and those with severe comorbidity and prior open surgery, which resulted in the differences presented in Table 1 . The higher morbidity rates, readmission and reoperation rates, as well as the prolonged hospital stay in the open group are likely a result of the former.

\section{Incisional hernia}

During follow-up, 24/170 (24\%) patients were diagnosed with IH after LARHC. The conversion rate was $16 \%$ (27/ 170). Five of the IHs occurred in the 27 converted patients (19\%), which was not different from the $19 \mathrm{IHs}$ in the 134 patients without conversion $(13 \%, P=0.546)$. Only four of these IH required surgical correction, and all others were asymptomatic or managed solely with supportive measures such as a hernia belt. In the open group, IH rate was $15 / 64$ (24\%) in the open group $(P=0.114)$ and only one patient required surgical correction. The symptoms, treatment, and classification of incisional hernias were similar between the laparoscopic and open cohort (Table 2).

A notable difference in IH rates was observed between surgeons in both the LARHC and open group (Fig. 2). IH rates differed between the two most experiences surgeons (12/84 for surgeon $\mathrm{C}$ and $6 / 69$ for surgeon $\mathrm{D}, P=0.02$ ). The median duration until diagnosis of IH was 7 (4-12) versus 14 (1-42) months in the LARHC and open group, respectively $(P=0.700)$. Uni- and multivariable analysis to identify risk factors for IH is displayed in Table 3. A history of smoking, higher BMI, and surgical site infection were identified as risk factors for $\mathrm{IH}$, and interestingly, the laparoscopic approach was not protective.

\section{Discussion}

This study reports on the IH rates after LARHC in a single hospital where LARHC is the technique of choice and laparoscopic colorectal surgery is considered in every patient without clear contraindications. In this relatively unselected cohort, IH incidence was 24/170 (14\%), while only $4(2 \%)$ patients required operative correction of the IH.

Data on IH after LARHC or LRHC are inconsistent in the literature; they primarily come from small series with few events and series from centers using several laparoscopic techniques. A comparative study of LRHC with LARHC found only one case of IH in the LARHC group out of a total 72 patients [18], while a similar study diagnosed six IHs in 23 LARHC cases compared to none in 21 LRHC cases [18]. A larger study found IHs in 2 out of 91 LRHC patients and 17 out of 100 LARHC patients [19]. Several factors might contribute to the large variation in IH incidence in these LARHC cohorts ranging from 4 to $21 \%$. Firstly, the definitions of IH vary, as does the reported duration of follow-up, and as mentioned above, the series include only 29-100 LARHC cases. In addition, these three centers performed LRHC and LARHC in similar proportions in the study period, which might result in differences compared to a center dedicated to either LARHC or RHC.

The present study was performed in a center in which laparoscopy is considered the standard for all colectomy procedures in all patients, unless there is a valid reason to consider otherwise, such as emergency procedures or $\mathrm{T} 4$ tumors. LARHC has been the standard RHC approach for all surgeons, and therefore, considerable experience has been accumulated, which is reflected in the mean duration of $109 \mathrm{~min}$ of LARHC procedures, compared to 142-186 min in other reports [18-20] and the low incidence of anastomotic leaks (2\%). 
Table 1 Clinical characteristics

\begin{tabular}{|c|c|c|c|c|}
\hline & All patients $(n=234)$ & Laparoscopy-assisted $(n=170)$ & $\begin{array}{l}\text { Open } \\
(n=64)\end{array}$ & $P$ value \\
\hline Age [median (IQR)] & $73(66-79)$ & $72(67-79)$ & $74(66-81)$ & 0.511 \\
\hline Male gender $[n(\%)]$ & $113(48)$ & $80(47)$ & $33(52)$ & 0.560 \\
\hline BMI [median (IQR)] & $25(23-29)$ & $26(23-29)$ & $24(22-27)$ & 0.017 \\
\hline ASA score $[n(\%)]$ & & & & 0.050 \\
\hline I & $34(15)$ & 29 (17) & $5(8)$ & \\
\hline II & $138(59)$ & $102(60)$ & $26(56)$ & \\
\hline III & $61(28)$ & $39(23)$ & $22(34)$ & \\
\hline IV & $1(0)$ & - & $1(2)$ & \\
\hline Previous abdominal surgery $[n(\%)]$ & $69(30)$ & $47(28)$ & $22(34)$ & 0.337 \\
\hline Emergency surgery $[n(\%)]$ & $25(11)$ & $1(1)$ & $24(38)$ & $<0.001$ \\
\hline T stage $[n(\%)]$ & & & & $<0.001$ \\
\hline 0 or in situ & $6(3)$ & $6(4)$ & - & \\
\hline I & $11(5)$ & $11(7)$ & - & \\
\hline II & $34(15)$ & $30(18)$ & $4(6)$ & \\
\hline III & $150(64)$ & $108(64)$ & $42(66)$ & \\
\hline IV & $33(14)$ & $15(9)$ & $18(28)$ & \\
\hline$N$ stage $[n(\%)]$ & & & & 0.262 \\
\hline I & $58(25)$ & $38(22)$ & $20(31)$ & \\
\hline II & $36(15)$ & $25(15)$ & $11(17)$ & \\
\hline M1 stage $[n(\%)]$ & $27(12)$ & $13(20)$ & $14(8)$ & 0.020 \\
\hline Operating time [min, median (IQR)] & $107(85(133)$ & $109(88-135)$ & $99(79-129)$ & 0.091 \\
\hline Conversion $[n(\%)]$ & - & $27(16)$ & - & - \\
\hline Major morbidity $[\geq$ Dindo IIIA, $n(\%)]$ & $27(12)$ & $10(6)$ & $17(27)$ & $<0.001$ \\
\hline CCI [median (IQR)] & $0(0-21)$ & $0(0-21)$ & $21(0-35)$ & $<0.001$ \\
\hline Incisional hernia $[n(\%)]$ & $39(17)$ & $24(14)$ & $15(24)$ & 0.114 \\
\hline Asymptomatic & $22(9)$ & $11(6)$ & $11(17)$ & \\
\hline Symptomatic; non-operative management & $12(5)$ & $9(5)$ & $3(5)$ & \\
\hline Symptomatic; operative correction & $5(2)$ & $4(2)$ & $1(2)$ & \\
\hline Surgical site infection $[n(\%)]$ & $26(15)$ & $22(13)$ & $14(22)$ & 0.105 \\
\hline Anastomotic leak $[n(\%)]$ & $7(3)$ & $3(2)$ & $4(6)$ & 0.091 \\
\hline Readmissions $[n(\%)]$ & $21(9)$ & $11(7)$ & $10(16)$ & 0.039 \\
\hline Reoperation $[n(\%)]$ & $15(6)$ & $5(3)$ & $10(16)$ & 0.001 \\
\hline Hospital stay [days, $n(\%)$ ] & $9(7-13)$ & $8(7-9)$ & $14(9-19)$ & $<0.001$ \\
\hline 90-day mortality $[n(\%)]$ & $4(2)$ & $1(1)$ & $3(5)$ & 0.063 \\
\hline
\end{tabular}

Interestingly, there was a notable difference in $\mathrm{IH}$ rates after LARHC between surgeons, especially between the most experiences surgeons C and D (25 versus 9\%). While many factors could be debit to the difference, surgeon D placed two absorbable standing fascia sutures in the caudal corner of the extraction site in addition to the running suture, while surgeon $\mathrm{C}$ used the running suture only. With the latter technique, the fascia may not be clearly visible during the final sutures, which might have led to suboptimal closure. Several studies concluded a continuous suture with longer suture to wound length ratios and small bites are effective to limit $\mathrm{IH}$ incidence, which could also have caused the differences observes in this report [21-23].

SSI was the most important risk factor for the development of IH. Proliferation of bacteria affects the wound healing process and leads to a decrease in the synthesis of collagen which reduces the strength of the abdominal wall and a higher risk of dehiscence [24, 25]. Therefore, all efforts to reduce SSI could result in a lower incidence of IH. The other risk factors in this study BMI and history of smoking, or age as reported in the literature are less easily or impossible to influence. 
Table 2 Comparison of hernia symptoms, management, and classification in the laparoscopy-assisted and open group

\begin{tabular}{lll}
\hline & Laparoscopy-assisted $(n=24)$ & Open $(n=15)$ \\
\hline Hernia symptoms and treatment & & $P$ value \\
Asymptomatic & $11(46)$ & $11(73)$ \\
Symptomatic; non-operative management & $9(38)$ & $3(20)$ \\
Symptomatic; operative correction & $4(17)$ & $1(7)$ \\
Hernia classification & & $1(7)$ \\
M2-W1 & - & $1(7)$ \\
M3-W1 & $4(17)$ & $10(67)$ \\
M3-W2 & $15(63)$ & $2(13)$ \\
M3-W3 & $1(8)$ & - \\
M5-W1 & $1(8)$ & $1(7)$ \\
L2-W1 & $3(13)$ & 0.435 \\
\hline
\end{tabular}

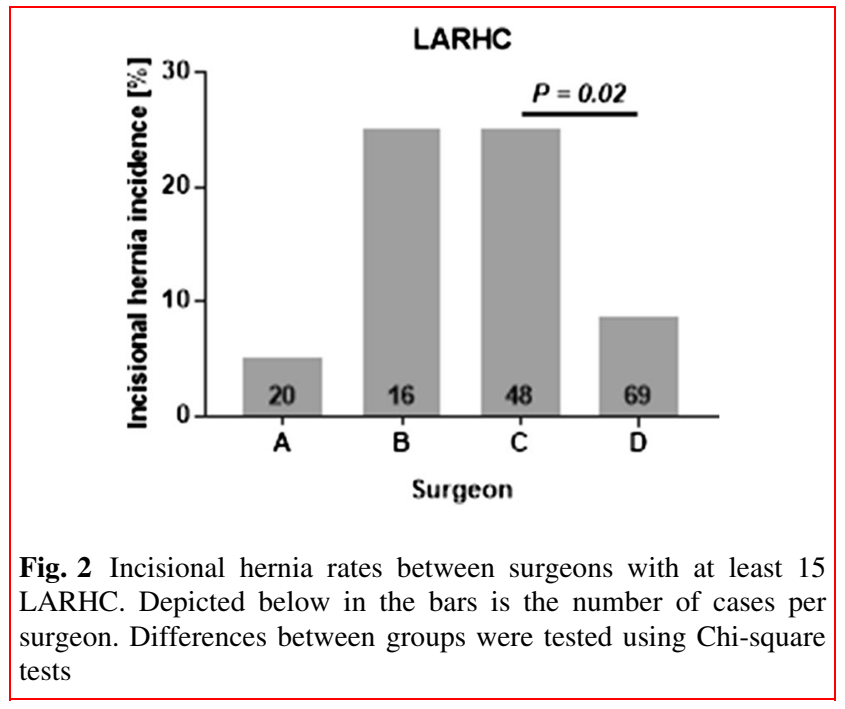

Different approaches for laparoscopic RHC have distinct advantages and disadvantages; these differences limit their comparison. LARHC requires a less operative time compared to LRHC and is a less demanding laparoscopic technique, which potentially reduces conversion rates and anastomotic leaks [26, 27]. The benefit of LRHC is the freedom of extraction site, which can be any incision such as a Pfannenstiel incision [21, 28]. This could reduce the IH rate $[19,20]$. Although numerous studies have compared different techniques, most are small and retrospective and focus on a specific outcome, which results in heterogeneous results often without large differences between techniques. Also the surgeon experience with the technique is underreported, and often several techniques are performed within a single center. This report demonstrates the results of a dedicated laparoscopic colorectal surgery center, with LARHC as primary technique for RHC.

Table 3 Uni- and multivariable analysis of factors contributing to the development of incisional hernia after LARHC

\begin{tabular}{|c|c|c|c|c|}
\hline & \multicolumn{2}{|l|}{ Univariable analysis } & \multicolumn{2}{|l|}{ Mulitvariable analysis } \\
\hline & Odds ratio $(95 \% \mathrm{CI})$ & $P$ value & Odds ratio $(95 \% \mathrm{CI})$ & $P$ value \\
\hline Age & $0.99(0.96-1.02)$ & 0.548 & & \\
\hline Male gender & $1.68(0.84-3.37)$ & 0.146 & & \\
\hline BMI & $1.07(1.00-1.15)$ & 0.048 & $1.08(1.00-1.16)$ & 0.043 \\
\hline History of smoking & $1.99(0.98-4.02)$ & 0.056 & $2.14(1.03-4.41)$ & 0.040 \\
\hline Steroid use & $0.71(0.23-2.16)$ & 0.548 & & \\
\hline Metastatic disease & $1.51(0.57-4.02)$ & 0.413 & & \\
\hline Previous abdominal surgery & $1.08(0.51-2.27)$ & 0.847 & & \\
\hline Laparoscopic approach & $0.54(0.26-1.11)$ & 0.091 & & \\
\hline ASA III-IV & $1.49(0.83-3.12)$ & 0.291 & & \\
\hline Surgical site infection & $2.67(1.18-6.03)$ & 0.018 & $2.99(1.28-7.00)$ & 0.012 \\
\hline
\end{tabular}


This study has several limitations consequential to its retrospective design. IH may not always be symptomatic, and the incidence could be missed during physical examination. However, all patients have a follow-up according to colorectal protocol with abdominal diagnostics, which were all reviewed, and it is unlikely that symptomatic IH would be missed during follow-up. Also the open group was shown to demonstrate patient selection and underscore the unselected LARHC group. Furthermore, the cohort is still relatively small and single center. However, compared with other studies, the present study has one of the largest LARHC patient populations reported. Also to our knowledge, this is the first study on IH in a cohort where laparoscopic-assisted RHC was the standard procedure for right-sided colorectal cancer.

In conclusion, the incidence of clinically relevant $\mathrm{IH}$ was low. The main risk factor associated with IH was SSI, although closing technique may also play an important role.

Different laparoscopic strategies for right hemicolectomy have different trade-offs. For a laparoscopic-assisted approach, the risk of IH should be taken into account in shared decision making. But it does not seem to outweigh possible advantages.

\section{Compliance with ethical standards}

Conflict of interest The authors declare that they have no conflict of interest.

Ethical standards The need for ethical approval and individual informed consent was waived by the institutional medical ethics committee.

Open Access This article is distributed under the terms of the Creative Commons Attribution 4.0 International License (http://crea tivecommons.org/licenses/by/4.0/), which permits unrestricted use, distribution, and reproduction in any medium, provided you give appropriate credit to the original author(s) and the source, provide a link to the Creative Commons license, and indicate if changes were made.

\section{References}

1. Braga M, Frasson M, Vignali A et al (2007) Open right colectomy is still effective compared to laparoscopy: results of a randomized trial. Ann Surg 246:1010-1014 (discussion 1014-1015)

2. Lacy AM, Garcia-Valdecasas JC, Delgado S et al (2002) Laparoscopy-assisted colectomy versus open colectomy for treatment of non-metastatic colon cancer: a randomised trial. Lancet 359:2224-2229

3. Ramacciato G, D'Angelo F, Aurello P et al (2008) Right hemicolectomy for colon cancer: a prospective randomised study comparing laparoscopic vs. open technique. Chir Ital 60:1-7
4. Rondelli F, Trastulli S, Avenia N et al (2012) Is laparoscopic right colectomy more effective than open resection? A metaanalysis of randomized and nonrandomized studies. Colorectal Dis 14:e447-e469

5. Weeks JC, Nelson H, Gelber S et al (2002) Short-term quality-oflife outcomes following laparoscopic-assisted colectomy vs open colectomy for colon cancer: a randomized trial. JAMA 287:321-328

6. Lacy AM, Delgado S, Castells A et al (2008) The long-term results of a randomized clinical trial of laparoscopy-assisted versus open surgery for colon cancer. Ann Surg 248:1-7

7. Jayne DG, Guillou PJ, Thorpe H et al (2007) Randomized trial of laparoscopic-assisted resection of colorectal carcinoma: 3-year results of the UK MRC CLASICC Trial Group. J Clin Oncol 25:3061-3068

8. Clinical Outcomes of Surgical Therapy Study G, Nelson H, Sargent DJ et al (2004) A comparison of laparoscopically assisted and open colectomy for colon cancer. $N$ Engl $J$ Med 350:2050-2059

9. Wang G, Zhou J, Sheng W et al (2017) Hand-assisted laparoscopic surgery versus laparoscopic right colectomy: a metaanalysis. World J Surg Oncol 15:215

10. Aalbers AG, Biere SS, van Berge Henegouwen MI et al (2008) Hand-assisted or laparoscopic-assisted approach in colorectal surgery: a systematic review and meta-analysis. Surg Endosc 22:1769-1780

11. Cirocchi R, Trastulli S, Farinella E et al (2013) Intracorporeal versus extracorporeal anastomosis during laparoscopic right hemicolectomy-systematic review and meta-analysis. Surg Oncol 22:1-13

12. van Oostendorp S, Elfrink A, Borstlap W et al (2017) Intracorporeal versus extracorporeal anastomosis in right hemicolectomy: a systematic review and meta-analysis. Surg Endosc 31:64-77

13. Kisielinski K, Conze J, Murken AH et al (2004) The Pfannenstiel or so called "bikini cut": still effective more than 100 years after first description. Hernia 8:177-181

14. Van Leersum NJ, Snijders HS, Henneman D et al (2013) The Dutch surgical colorectal audit. Eur J Surg Oncol 39:1063-1070

15. Muysoms FE, Miserez M, Berrevoet F et al (2009) Classification of primary and incisional abdominal wall hernias. Hernia 13:407-414

16. Dindo D, Demartines N, Clavien PA (2004) Classification of surgical complications: a new proposal with evaluation in a cohort of 6336 patients and results of a survey. Ann Surg 240:205-213

17. Slankamenac K, Graf R, Barkun J et al (2013) The comprehensive complication index: a novel continuous scale to measure surgical morbidity. Ann Surg 258:1-7

18. Roscio F, Bertoglio C, De Luca A et al (2012) Totally laparoscopic versus laparoscopic assisted right colectomy for cancer. Int J Surg 10:290-295

19. Shapiro R, Keler U, Segev L et al (2016) Laparoscopic right hemicolectomy with intracorporeal anastomosis: short- and longterm benefits in comparison with extracorporeal anastomosis. Surg Endosc 30:3823-3829

20. Vergis AS, Steigerwald SN, Bhojani FD et al (2015) Laparoscopic right hemicolectomy with intracorporeal versus extracorporeal anastamosis: a comparison of short-term outcomes. Can J Surg 58:63-68

21. Israelsson LA, Jonsson T (1993) Suture length to wound length ratio and healing of midline laparotomy incisions. Br J Surg 80:1284-1286

22. Diener MK, Voss S, Jensen K et al (2010) Elective midline laparotomy closure: the INLINE systematic review and metaanalysis. Ann Surg 251:843-856 
23. Deerenberg EB, Harlaar JJ, Steyerberg EW et al (2015) Small bites versus large bites for closure of abdominal midline incisions (STITCH): a double-blind, multicentre, randomised controlled trial. Lancet 386:1254-1260

24. Itatsu K, Yokoyama Y, Sugawara G et al (2014) Incidence of and risk factors for incisional hernia after abdominal surgery. Br J Surg 101:1439-1447

25. Sorensen LT, Hemmingsen UB, Kirkeby LT et al (2005) Smoking is a risk factor for incisional hernia. Arch Surg 140:119-123

26. Jamali FR, Soweid AM, Dimassi H et al (2008) Evaluating the degree of difficulty of laparoscopic colorectal surgery. Arch Surg 143:762-767 (discussion 768)
27. Vignali A, Bissolati M, De Nardi P et al (2016) Extracorporeal vs. intracorporeal ileocolic stapled anastomoses in laparoscopic right colectomy: an interim analysis of a randomized clinical trial. J Laparoendosc Adv Surg Tech A 26:343-348

28. Benlice C, Stocchi L, Costedio MM et al (2016) Impact of the specific extraction-site location on the risk of incisional hernia after laparoscopic colorectal resection. Dis Colon Rectum 59:743-750

Publisher's Note Springer Nature remains neutral with regard to jurisdictional claims in published maps and institutional affiliations. 